\title{
Charge Density Mapping via Scanning Diffraction in Scanning Transmission Electron Microscopy
}

\author{
Christopher Addiego ${ }^{1}$, Wenpei $\mathrm{Gao}^{2}$, Xiaoqing $\operatorname{Pan}^{1,2,3}$ \\ ${ }^{1}$ Department of Physics and Astronomy, University of California - Irvine, Irvine, CA 92697 , \\ USA \\ ${ }^{2}$ Department of Chemical Engineering and Materials Science, University of California - Irvine, \\ Irvine, CA 92697, USA \\ ${ }^{3}$ Irvine Materials Research Institute, University of California - Irvine, Irvine, CA 92697, USA
}

The redistribution of electrons to form chemical bonds is a fundamental process which determines the characteristics of materials; many materials are classified based on the types of bonds that they form. Therefore, the measurement of electron charge density is an important step in building structure-property relationships. The theory of bonding and orbital hybridization is well understood and, as a result, first principles calculations can provide highly detailed charge density maps. However experimental measurement of charge density is still not routine. Various techniques using X-ray diffraction and convergent beam electron diffraction (CBED) have been developed to study charge density, but they require very involved simulation and analysis of diffraction data from the material under study. Quantitative CBED (QCBED) is one such example where experimental CBED patterns must be fit to simulated CBED patterns before determining the charge density [1]. This methodology of fitting experimental data to simulation maps fine details of the three-dimensional charge density but is difficult to apply to irregular structures like defects and interfaces in materials because of the limitation in spatial resolution, and the increase of computation time and complexity in pattern fitting.

Scanning diffraction provides one such avenue for directly imaging charge density in scanning transmission electron microscope (STEM) without the need to fit with simulated data. Similar to QCBED, scanning diffraction is based on 4D STEM datasets captured on a high-speed pixelated detector that is synchronized to the scanning electron probe. Based on work done by [2], the center of intensity in a diffraction pattern is equivalent to the average momentum transfer and the momentum transfer is inversely proportional to the electric field in the material. With the map of projected electric field in two-dimension (2D), calculating the divergence of the measured 2D electric field yields the local charge density projected along the beam direction. In this paper, we present results from a study of electric field and charge density in $\mathrm{SrTiO}_{3}$ and $\mathrm{BiFeO}_{3}$.

Scanning diffraction data was collected using Gatan OneView camera on a Jeol ARM300F STEM at $300 \mathrm{kV}$, with the acquisition speed of 300 fps. Electric field maps from samples of $\mathrm{SrTiO}_{3}$ and $\mathrm{BiFeO}_{3}$ with sub-angstrom resolution are shown in Figure 1. At the interface, results show how charge density changes across a ferroelectric-insulator interface in Figure 2. In $\mathrm{SrTiO}_{3}$, both the electric field and charge density around each atom are rotationally symmetric. In $\mathrm{BiFeO}_{3}$, we observed an asymmetric electric field pointed preferentially along the diagonal, which corresponds to the polarization along (111) in $\mathrm{R}-$ like $\mathrm{BiFeO}_{3}$ [3], and distortions to the charge density. 
With a real space charge density imaged with atomic resolution, structure information such as the position of light elements, such as the oxygen atoms in $\mathrm{SrTiO}_{3}$ and $\mathrm{BiFeO}_{3}$ can be revealed immediately. Especially, the tilt of the oxygen octahedron caused by the polarization in $\mathrm{BiFeO}_{3}$ is visible as an elongation of the oxygen columns. The possibilities offered by the scanning diffraction including measuring the polarized electric field in the electric field map and quantifying the separation of the positive and negative charges will be discussed.

\section{References:}

[1] P. N. H. Nakashima, et al., Science 311 (2011), 1583-1586.

[2] K. Müller-Caspary, et al., Ultramicroscopy 178 (2016), 62-80

[3] C.T. Nelson, et al., Nano Letters 11 (2011), 828-824

[4] This work was supported by the Department of Energy (DOE) under Grant DE-SC0014430. TEM experiments was conducted using the facilities in the Irvine Materials Research Institute (IMRI) at the University of California-Irvine.

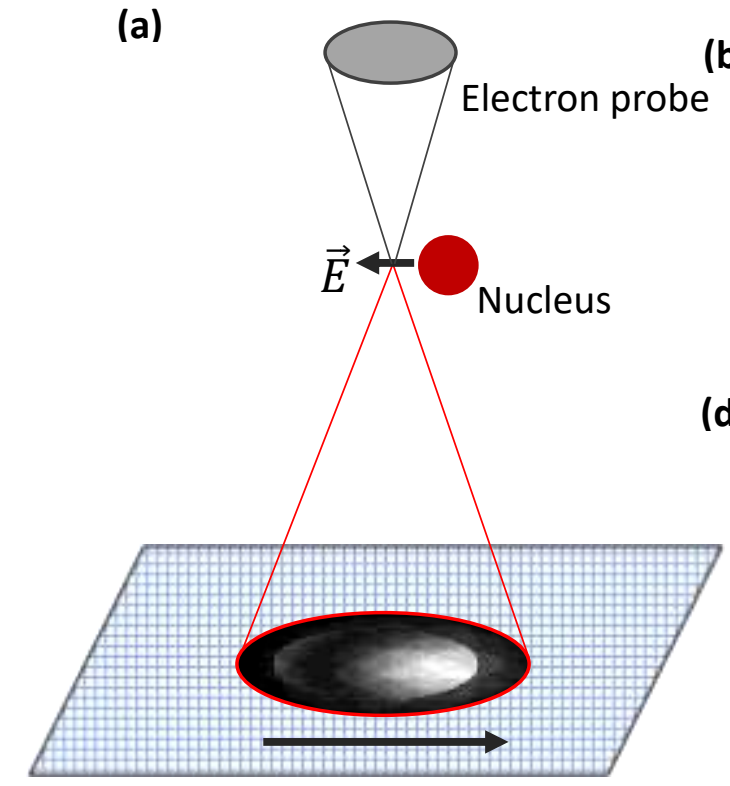

Pixelated detector (b)

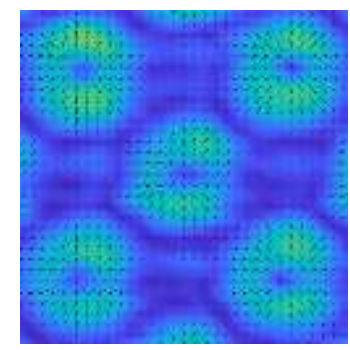

(d)

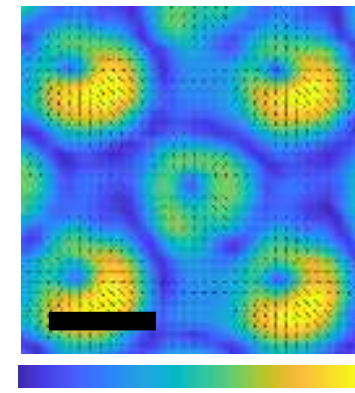

Electric field magnitude (c)

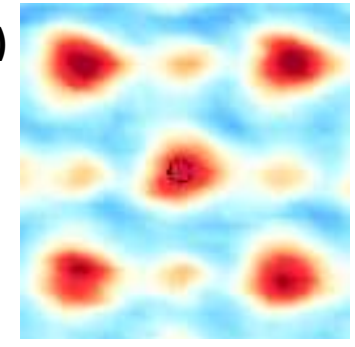

(e)

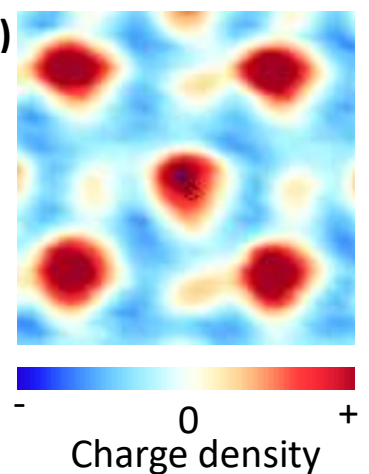

Figure 1 (a) A schematic depicting the set up for 4D STEM with a pixelated detector. (b) and (c) show the electric field and charge density in $\mathrm{SrTiO}_{3}$ unit cell. (d) and (e) show the electric field and charge density in a $\mathrm{BiFeO}_{3}$ unit cell. The scale bar is $2 \AA$.

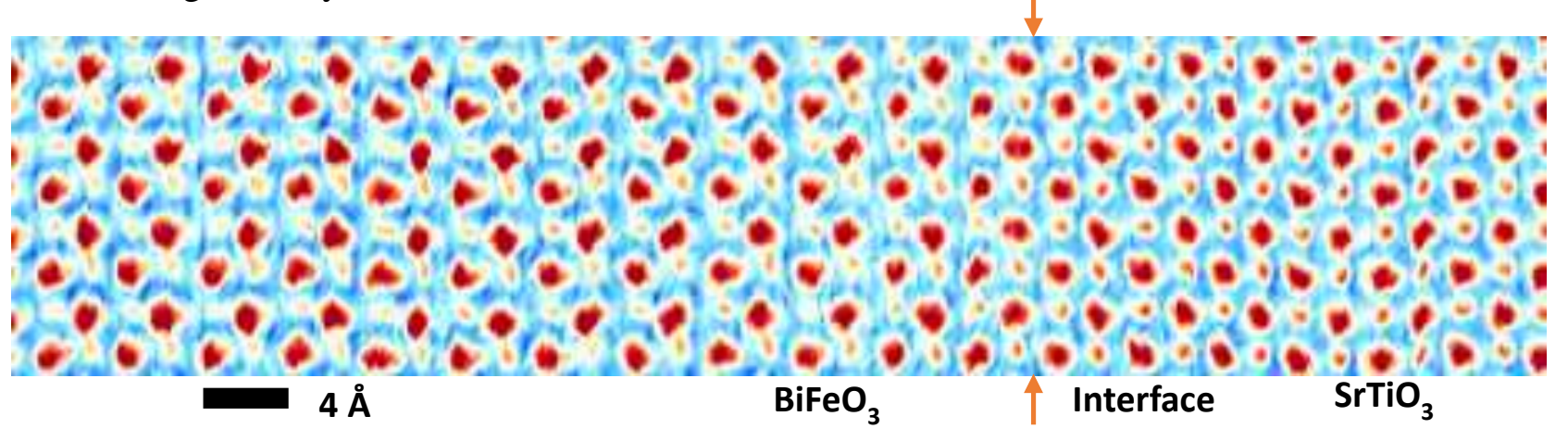

Figure 2 The charge density image of a $\mathrm{BiFeO}_{3}-\mathrm{SrTiO}_{3}$ interface. Polarization of the $\mathrm{Fe}$ atom is towards the upper right. 